$\begin{array}{cl}\begin{array}{c}\text { Revue } \\ \text { de } / \text { histoire }\end{array} & \text { Revue de l'histoire des religions } \\ \text { des religions } & \begin{array}{l}2 \mid 2009 \\ \text { Varia }\end{array}\end{array}$

Comparatisme orienté et étymologie comparée chez Max Müller : l'équation Bráhman = Verbum

Ideological leanings and comparative etymology in Max Müller, the equation Bráhman $=$ Verbum

Guillaume Ducœur

(2) OpenEdition

Journals

Édition électronique

URL : http://journals.openedition.org/rhr/7227

DOI : $10.4000 /$ rhr.7227

ISSN : 2105-2573

Éditeur

Armand Colin

Édition imprimée

Date de publication : 1 avril 2009

Pagination : 163-180

ISBN : 978-2200-92590-1

ISSN : 0035-1423

Référence électronique

Guillaume Ducœur, « Comparatisme orienté et étymologie comparée chez Max Müller : l'équation

Bráhman = Verbum », Revue de l'histoire des religions [En ligne], 2 | 2009, mis en ligne le 01 avril 2012, consulté le 02 mai 2019. URL : http://journals.openedition.org/rhr/7227 ; DOI : 10.4000/rhr.7227

Tous droits réservés 


\section{Comparatisme orienté et étymologie comparée chez Max Müller : l'équation Bráhman $=$ Verbum}

Au cours de sa vie, Max Müller (1823-1900) a plus d'une fois tenté de démontrer l'unité de pensée entre stoïciens, néo-platoniciens, chrétiens et védantistes par la mise en équation de bráhman = verbum. L'approche étymologique qu'il définissait comme "la Science de la Vérité » devait prouver que du Levant au Ponant, les philosophes avaient abouti naturellement aux mêmes conclusions cosmogoniques : le monde phénoménal fut

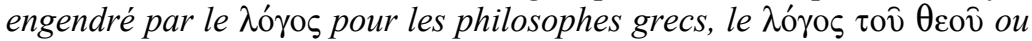
verbum dei pour les chrétiens ou le bráhman pour les rși et les philosophes védantistes. La présente recherche vise à s'interroger sur le contexte historique d'une telle démonstration, les motivations sous-jacentes et les procédés méthodologiques de son auteur.

\section{Ideological leanings and comparative etymology in Max Müller, the equation Bráhman $=$ Verbum}

More than once in the course of his life, Max Müller (1823-1900) sought to show the unity of thought between the Stoics, neo-Platonists, Christians and Vedantists by means of the equation : bráhman = verbum. For Müller, the etymological approach or "the Science of Truth", showed that from East to West, philosophers naturally came to the same conclusions regarding the origins of creation : the phenomenal world was born

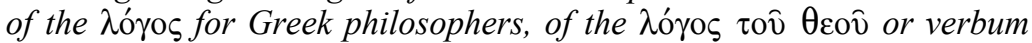
dei for Christians and of the bráhman for rși and Vedantist philosophers. This paper looks into the historical context of this position, the underlying motives and the methodological processes of the author. 
La recherche étymologique comme connaissance de la nature des choses et à travers cette dernière de l'Être, remonte haut dans l'Antiquité. Du Cratyle de Platon à l'œuvre wartburgienne, en passant par les Étymologies d'Isidore de Séville ou les lexiques byzantins, l'étymologie s'est imposée, sans pourtant y être jamais arrivée, comme une "science exacte» du langage, suivant l'étymologie même de ce néologisme stoïcien. Il n'est donc pas étonnant que l'étymologie soit venue au cours des siècles appuyer de nombreuses théories et plus particulièrement lors de la découverte, $\mathrm{du} \mathrm{XVII}^{\mathrm{e}}$ au $\mathrm{XIX}^{\mathrm{e}}$ siècle, de langues encore inconnues des Européens. L'étymologie apparaissait comme l'indispensable outil permettant de saisir le sens premier d'un mot et surtout, pour certaines disciplines, le garant scientifique de toute approche comparative des civilisations et de leurs religions. Selon les visées de chaque domaine d'application, l'étymologie a été usitée à des fins bien spécifiques et a parfois servi des idéologies tout orientées. Ainsi, au début du XVIII ${ }^{\mathrm{e}}$ siècle, par exemple, Jean-Venant Bouchet, missionnaire jésuite à Maduré, affirmait par analogies phonétique et morphologique que brahmā n'était autre qu'une déformation d'Abraham tout comme sa parèdre Sarasvatî, celle de son épouse Sarah ${ }^{1}$, confirmant ainsi la croyance des Pères de l'Eglise selon laquelle les Indiens descendaient des fils de Noé. Eloignés de la Palestine, berceau du judaïsme ancien, la loi mosaïque, que ces Indiens auraient reçue au cours de leur histoire, et le souvenir lointain d'us et coutumes ancestraux auraient été sujets à une lente dégénérescence sur leur sol$^{2}$. Par des analogies linguistiques, notamment en décomposant arbitrairement les phonèmes, comme le faisait déjà à outrance un siècle auparavant

1. «Il est visible que de Brama à Abraham il n'y a pas beaucoup de chemin à faire; et il serait à souhaiter, que nos savants, en matière d'étymologies, n'en eussent point adoptées de moins raisonnables, et de plus forcées. ", Lettre du Père Bouchet, de la Compagnie de Jésus, Missionnaire de Maduré, \& Supérieur de la nouvelle Mission de Carnate, à Monseigneur l'ancien évêque d'Avranches, in Cérémonies et coutumes religieuses des peuples idolâtres, représentées par des figures dessinées de la main de Bernard Picart : avec une explication historique et quelques dissertations curieuses, tome second, première partie, Amsterdam, 1728, p. 102.

2. Guillaume Ducœur, Brahmanisme et encratisme à Rome au III siècle après J.-C., Paris, L’Harmattan, 2001. 
Samuel Bochart dans sa Geographia Sacra, les savants d'alors pensaient pouvoir retrouver dans les sources sanskrites les vestiges de leur propre tradition judéo-chrétienne.

L'histoire comparée des religions, en tant que discipline scientifique née au cours du XIX ${ }^{\mathrm{e}}$ siècle, n'a pas dérogé à la règle et a largement usé de l'étymologie afin de justifier certaines de ses thèses. Dans le tumulte des nouvelles théories énoncées par le darwinisme et l'évolutionnisme, des méthodes classificatoires des espèces en botanique et en biologie, des découvertes sans précédent de la grammaire comparée, des études anthropologiques et sociologiques, l'histoire comparée des religions, en s'inspirant de leur méthode comparative respective, espérait découvrir enfin l'origine commune des croyances et remonter ainsi à la pensée religieuse primitive de nos lointains ancêtres. Très impressionné dès son plus jeune âge par la grammaire comparée des langues indo-européennes ${ }^{3}$, Max Müller a, tout au long de sa vie, toujours considéré comme fondamentale la recherche étymologique qu'il définissait comme étant «la Science de la Vérité »" C'est par ce biais et la mise en équation bráhman = verbum qu'il tenta de démontrer l'unité de pensée entre stoïciens, néo-platoniciens, chrétiens et védantistes. Par cette tentative d'équivalence, Max Müller essaya de prouver que les philosophes grecs, et à leur suite les premiers Pères de l'Eglise, tout comme les sages de l'Inde avaient abouti naturellement aux mêmes conclusions cosmogoniques : le monde phénoménal aurait été engendré par le $\lambda$ óros pour les philosophes grecs, le $\lambda$ ó ou le bráhman pour les rṣ̣i puis les philosophes védantistes. La présente étude vise donc à s'interroger non seulement sur le contexte historique d'une telle démonstration mais encore sur les procédés méthodologiques employés par Max Müller. Nous nous prononcerons donc non pas sur la valeur d'une telle équation mais bel et bien sur la mise en équation elle-même, sur la méthode utilisée, sur le recours à l'étymologie et à l'histoire comparée des religions,

3. Max Müller, Nouvelles études de mythologie, in Mythologie comparée, édition établie, présentée et annotée par P. BRUNEL, Paris, R. Laffont, 2002, p. 417418.

4. M. Müller, Nouvelles leçons sur la science du langage, tome premier, Phonétique et étymologie, traduit de l'anglais par Georges Harris et Georges Perrot, Paris, A. Durand et Pedone-Lauriel, 1867, p. 307. 
mises, toutes deux, au service d'un comparatisme orienté à fins personnelles.

Il n'est nullement besoin ici de présenter la vie et l'œuvre considérable de Max Müller (1823-1900), son implication dans la diffusion des traductions de sources orientales et plus particulièrement sanskrites, ni l'influence de son approche linguistique de la mythologie comparée au cours de la seconde moitié du XIX ${ }^{\mathrm{e}}$ siècle ${ }^{5}$. Malgré des avancées certaines, ni sa théorie de la maladie du langage, ni celle du solarisme, ni même celle de l'hénothéisme védique ${ }^{6}$ n'ont pu tenir devant la critique des mythologues, des indianistes et surtout des linguistes qui, après avoir démontré l'irrecevabilité de telles thèses, n'ont pas manqué de discréditer l'approche naturaliste de l'École d'Oxford. Mais notre propos n'a aucun caractère généralisant. En d'autres termes, ce que nous allons exposer sur la démarche de Max Müller ne vaut que dans les limites de cette équation étymologique. D'ailleurs, il serait incorrect de prétendre que ce grand érudit n'ait pas lui-même émis parfois quelques réserves sur les résultats de ses propres recherches.

\section{Max Müller, un védantiste Convaincu}

L'équation bráhman $=$ verbum est exposée, dans sa forme la plus synthétique ${ }^{7}$, dans la troisième conférence, "Similarities and Differences between Indian and European Philosophy », que Max Müller prononça en mars 1894 à l'Institut Royal de GrandeBretagne. Elle fait donc partie d'une longue communication sur la philosophie védantique qui fut publiée la même année ${ }^{8}$ puis, dans

5. Sur la vie de Max Müller et son approche linguistique de l'indo-européen et des langues sémitiques, on se reportera avec intérêt aux ouvrages de Lourens Peter van den Bosch, Friedrich Max Müller : a life devoted to the humanities, Leiden, Brill, 2002 et de Maurice Olender, Les langues du Paradis, édition revue et augmentée, Paris, Seuil, 2002, p. 157-175.

6. Voir la réfutation de William Dwight Whitney, « Le prétendu hénothéisme du Véda », Revue de l'Histoire des Religions, $3^{\mathrm{e}}$ année, tome sixième, Paris, E. Leroux, 1882, p. 129-143.

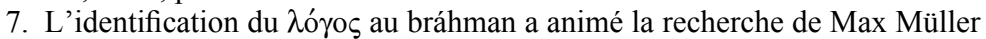
durant de longues années et ce jusqu'à sa mort. Son plus long développement se trouve consigné dans son ouvrage Theosophy or Psychological Religion, the Gifford Lectures, London, 1893.

8. M. Müller, Three Lectures on the Vedanta Philosophy, London, 1894. 
sa version française, en $1899^{9}$. Le titre de cette troisième conférence est significatif. La méthode comparative annoncée se veut être impartiale et prendre en considération tout autant les convergences que les divergences entre les deux objets d'étude. Or ces derniers, philosophies indienne et européenne, représentent des champs de recherche si vastes que nous sommes en droit de nous interroger sur le sens à leur donner. Ils trahissent cet optimisme et cette mégalomanie qui caractérisent si bien les érudits du XIX ${ }^{\mathrm{e}}$ siècle enthousiasmés par tant de découvertes. N'est-ce pas Max Müller lui-même qui prétendait dès 1870 qu' « établir une science des religions qui reposera sur la comparaison de toutes les religions de l'humanité, ou au moins des plus importantes, ce n'est plus là qu'une question de temps ». Paradoxe notoire de la démarche comparative qui se devrait de prendre en considération l'ensemble des objets d'étude et qui ne le peut guère dans la pratique, devant alors se contenter simplement des objets d'étude les plus caractéristiques. Or, les choix opérés ne peuvent être justifiés, car faire le choix de certaines religions au détriment d'autres relève d'un jugement de valeur. Quelles sont donc ces religions que Max Müller considère comme importantes? Probablement celles qui comptent le plus grand nombre de croyants à travers le monde, pensons aux trois monothéismes, ou qui ont quelque importance de par leurs sources scripturaires comme le védisme. A ceci s'ajoute la dichotomie Orient / Occident, Inde / Europe. Opposition géographique certes, mais aussi idéologique et religieuse. Max Müller compte-t-il effacer les distances, estomper les clivages qui séparent et opposent sur bien des points ces deux grands ensembles culturels? Fasciné par l'Inde, n'est-il pas celui qui s'est acculturé tout en demeurant dans sa propre culture? N'est-il pas l'homme tiraillé entre deux pensées qu'il souhaite n'être qu'une? Il y a dans son comparatisme une rencontre évidente qui trahit tant son indocentrisme que son européocentrisme.

Max Müller ne peut faire fi des différences manifestes entre les systèmes philosophiques grecs, chrétiens et indiens. Mais, il nous semble que de les souligner donne à sa démonstration étymologique encore plus de pertinence. La mise en équation doit,

9. M. Müller, Introduction à la philosophie du védanta, Paris, E. Leroux, 1899. 
dans la pensée müllérienne, permettre de passer outre les divergences et de retrouver au-delà de la spéculation philosophique de chacun des milieux intellectuels, produits de leur propre histoire culturelle, une origine commune de pensée. Le savant, en effet, n'accorde aucun crédit à l'hypothèse selon laquelle les Indiens auraient enseigné leur doctrine upanișadique aux Grecs aux temps de la conquête d'Alexandre le Macédonien. Pour lui, le témoignage d'Onésicrite relatant l'impossibilité de transmettre, à cause de la chaîne des traductions successives nécessaires, toute doctrine philosophique d'un milieu à un autre, sans en perdre l'essence, « montre combien il serait difficile d'admettre une influence exercée par $l^{\prime}$ 'Inde sur les philosophes grecs $»^{10}$. Si donc ressemblances il y a, elles se situent pour Max Müller à un niveau autre et non celui d'hypothétiques contacts et influences historiques entre la Grèce et l'Inde. La démarche historique laisse place alors à l'approche étymologique et, à partir de cette dernière, à la définition du sens à donner aux termes philosophiques comparés. En 1899, reprenant pour la énième fois l'équation bráhman = verbum, il affirmera alors que cette communauté linguistique ne peut être que le reflet d'une communauté de pensée et donc d'une pensée philosophique aryenne commune avant même la séparation ${ }^{11}$. Ainsi, passant outre l'étude historique des milieux philosophiques, Max Müller cherche avant tout à retrouver en l'Homme, quelle que soit l'époque à laquelle et le milieu dans lequel il vécut, une pensée unique du Divin. Cette motivation qui a pour une part largement contribué à l'orientation de ses recherches, est particulièrement bien exprimée tout au long des pages de son ouvrage Origine et développement de la religion étudiés à la lumière des religions de l'Inde dans lesquels il montre combien l'homme est de par nature foncièrement religieux et combien l'Occident, pris dans les controverses théologiques des Eglises chrétiennes de son temps, aurait tout intérêt à s'inspirer de la cohabitation indienne des expressions diverses de l'Infini. De même, lors de ces trois conférences, alors âgé de 71 ans, six ans avant sa mort, il ne peut s'empêcher de rappeler à son auditoire ce

10. M. Müller, La science du langage, cours professé à l'Institution royale de la Grande-Bretagne en l'année 1861, traduction de G. Harris et G. Perrot, Paris, A. Durand et Pedone-Lauriel, 1867, n. 1, p. 105.

11. M. Müller, Six Systems of Indian Philosophy; Samkhya and Yoga; Naya and Vaiseshika, London, 1899, p. 63. 
que Schopenhaüer lui-même avait dit au sujet de ses lectures des upanișad $^{12}$ et d'ajouter :

«Si ces paroles de Schopenhauer avaient besoin d'un endossement, je le donnerais volontiers comme le résultat de ma propre expérience, fruit d'une longue vie consacrée à l'étude de maintes philosophies et de maintes religions. Si l'on considère la philosophie comme une préparation à une bonne mort, ou euthanasie, je ne connais pas de meilleure préparation à cet effet que la philosophie védanta. $»^{13}$

Ces paroles sont d'importance si l'on veut saisir la visée première de Max Müller qui va rapprocher la notion grecque de $\lambda$ ó$_{0} \varsigma$, et notamment celle de la tradition chrétienne, de la pensée indienne védantique. Sa motivation première est en effet de mettre en parallèle christianisme et vedānta et de souligner ainsi une correspondance théologique évidente à son sens qui justifie, à elle seule, sa propre croyance en ce qu'il nomme à défaut de mieux l'Infini. La conclusion de ses Essais sur la science des religions est tout à fait parlante à cet égard :

«Bien que vivant sur le forum, et non plus dans la forêt, nous pouvons, à leur école, nous faire à différer du voisin, à aimer ceux qui nous haïssent pour nos convictions religieuses, ou à tout le moins nous pouvons désapprendre la haine et la persécution contre ceux dont les convictions, les espérances et les craintes, ou même les principes de morales, ne sont pas les nôtres. C'est là encore vivre en vānaprastha, c'est vivre une vie digne d'un vrai sage de la forêt, digne d'un homme qui sait ce que l'homme est, ce que la vie est, et qui a appris à se taire en face de l'Eternel et de l'Infini. ${ }^{14}$

Max Müller apparaît donc comme « celui qui sait », le vidvān des upaniṣad qui, ayant la connaissance de sa propre nature, celle de 1'ātman, peut alors se fondre en bráhman, le Principe suprême qui n'est pas sans rappeler, selon une épithète sanskrite commune, l'Infini de Max Müller. Faut-il dès lors admettre une orientation plus idéologique que scientifique de la part de ce mythologue? Son enthousiasme pour la philosophie védantique, sa tentative de vouloir à tout prix démontrer cette équation linguistique, ses hypo-

12. « Il n'existe pas dans le monde entier d'étude aussi profitable et aussi propre à élever l'esprit que celle des Oupanishads. Elle a été la consolation de ma vie, elle sera la consolation de ma mort », M. MüLLER, Introduction à la philosophie du védanta, Paris, E. Leroux, 1899, p. 10.

13. M. Müller, ibid., p. 10.

14. M. Müller, Origine et développement de la religion étudiés à la lumière des religions de l'Inde, Paris, C. Reinwald, 1873, p. 328. 
thèses d'une pensée théologique commune occidentale et orientale, nous portent à l'affirmer sans hésitation aucune. Et si nous avions encore besoin de le justifier, il nous suffirait de citer ce passage tiré de sa première conférence qui indique parfaitement l'ultime place qu'il conférait au vedānta au sein de son schéma évolutionniste des croyances religieuses de l'humanité et de celles de l'Inde, de l'hénothéisme à l'athéisme ou adévisme :

«C'est ce trésor de l'antique pensée religieuse que les sages des Oupanishads héritèrent de leurs ancêtres, et nous allons voir maintenant quel usage ils en firent, et comment ils découvrirent enfin la véritable relation qui existe entre ce que nous appelons le Divin ou l'Infini, tel qu'on le voit objectivement dans la nature, et le Divin ou Infini perçu subjectivement dans l'âme humaine. Nous serons alors plus à même de comprendre comment ils érigèrent sur cette antique base ce qui fut à la fois la philosophie la plus sublime et la religion la plus satisfaisante, le Védanta. $»^{15}$

Son comparatisme se doit donc d'être replacé dans son contexte, si nous voulons saisir au mieux cet acharnement linguistique que ses contemporains et ses successeurs ont dénoncé, rejeté et parfois même raillé. Partant du principe qu'il ne peut y avoir de séparation entre langage et pensée, il voit dans l'étymologie comparée l'assurance de saisir parfaitement ce que nos ancêtres, à travers leur expression langagière, avaient eux-mêmes perçu du Divin ou de l'Infini. Il est porté en cela par son époque et l'enthousiasme de la grammaire comparée des langues indo-européennes ainsi que des idéologies marquées comme celle de Schopenhaüer qui ira même jusqu'à tirer hors de ses origines judaïques, le christianisme et à n'y voir que l'aboutissement de la pensée indienne : « Le Nouveau Testament, au contraire, doit avoir une origine indoue quelconque; son éthique, qui transfère la morale dans l'ascétisme, son pessimisme et son avatar en témoignent. Tout cela le met en opposition décidée avec l'Ancien Testament. La doctrine chrétienne issue de la sagesse de l'Inde a recouvert le vieux tronc, complètement hétérogène pour elle, du grossier judaïsme $»^{16}$, allant parfois encore plus loin au sujet des échecs répétés d'évangélisation $\mathrm{du}$ peuple indien : «Nos religions ne prennent ni ne prendront

15. M. Müller, Introduction à la philosophie du védanta, Paris, E. Leroux, 1899, p. 34.

16. Raymond Schwab, La Renaissance Orientale, Paris, Payot, 1950, p. 450. 
racine dans l'Inde : la sagesse primitive de la race humaine ne se laissera pas détourner de son cours pour une aventure arrivée en Galilée $»^{17}$.

Après la découverte des sources sanskrites bouddhiques par Brian Hodgson et les travaux d'Eugène Burnouf sur les fondements de la doctrine bouddhique indienne, l'Inde apparut sous un nouveau visage. Le nirvāna, l'extinction de la soif d'existence, que les philosophes européens comprendront comme l'anéantissement de l'être, portera un coup fatal à la philosophie indienne en général. Dans son ouvrage L'Inde anglaise, son état actuel et son avenir, Jules Barthélemy-Saint-Hilaire, ancien ministre des Affaires étrangères de Jules Ferry déclarera que « [...] la sagesse indienne se réduit [...] à un génie poétique et religieux qui doit tenir une assez grande place dans les annales de l'esprit humain, sans être l'égale du génie grec, ni surtout l'égale du judaïsme. ${ }^{18}$ Le relais va donc être pris par d'autres milieux de pensée. Ainsi, par exemple, H. P. Blavatsky puisa largement dans ces recherches étymologiques et le balbutiement de ces études comparées entre philosophies grecque, chrétienne et védantique, afin d'étayer sa doctrine syncrétique parue en 1888 :

"Thus in Manu, Brahma (the Logos also) is shown dividing his body into two parts, male and female, and creating in the latter, who is Vach, Viraj, who is himself, or Brahma again - it is in this way a learned Vedantin Occultist speaks of that "goddess", explaining the reason why Eswara (or Brahma) is called Verbum or Logos; why in fact it is called Sabda Brahman."19

Au cours de cette même année, le colonel H. Olcott, l'un des fervents dirigeants de la Société théosophique, vint à Oxford dans le but de rencontrer Max Müller et de s'entretenir avec lui sur divers points concernant les religions indiennes. Leur entretien se prolongea par l'intermédiaire d'une correspondance continue au moins jusqu'en 1893. Mais bien que le savant oxfordien pût s'engager lui-même sur la voie difficile et exigeante du comparatisme entre religions de cultures différentes, il n'avait guère de considération

17. R. Schwab, ibid., p. 447-448.

18. Roger-Paul Droit, L'oubli de l'Inde, une amnésie philosophique, Paris, Seuil, 2004, p. 162.

19. Helena Petrovna Blavatsky, The Secret Doctrine. The Synthesis of Science, Religion, and Philosophy, vol. 1, 1888, p. 137. 
pour H. P. Blavatsky dont il dénonçait la méconnaissance des fondements des divers courants religieux de 1 ' $\operatorname{Inde}^{20}$.

Les conférences de Max Müller sur la philosophie védantique coïncident également avec le développement international de la mission Ramakrș̣na sous l'égide de Vivekananda. Ce dernier vint présenter la doctrine de son maitre et plus largement la philosophie védantique à Chicago au World's Congress of Religions lors de la World's Columbian Exposition de septembre 1893 avant de parcourir l'Europe et d'y faire un grand nombre de conférences. Là encore, Max Müller s'intéressa de près à cette grande figure mystique de l'Inde et alla même jusqu'à publier en 1898 un ouvrage sur la vie et l'enseignement védantique de Ramakrș̣na ${ }^{21}$ à partir de documents obtenus par le biais de ses disciples, notamment Vivekananda qu'il rencontra à Londres en mai 1896. Védantiste convaincu, Max Müller est intimement persuadé que "God is in all men, but all men are not in God : that is the reason why they suffer" 22 . En ces temps où la philosophie upanișadique, assombrie par la bouddhique, perdit tout attrait, la philosophie védantique, rendue vivante auprès des occidentaux par la bouche des disciples de Ramakṛṣna, apparaissait comme la mieux aboutie à rendre compte de la pensée indienne ancienne et contemporaine, la mieux préparée à forcer un passage entre Inde et Europe, une Europe qui se cherchait encore dans l'Inde.

\section{L'ÉTYMOLOGIE AU SECOURS D'UN COMPARATISME ORIENTÉ}

Pour parvenir à résoudre l'équation posée et à prouver les convergences entre philosophies indienne et européenne, Max Müller dut prendre des chemins détournés et démontrer qu'aux origines le neutre védique bráhman avait une signification autre que celle entendue par les brahmanes et que cette dernière ne renvoyait pas uniquement à la «formule sacrée $»^{23}$. Il a alors recours à des

20. L. P. van Den Bosch, Friedrich Max Müller: a life devoted to the humanities, Leiden, Brill, 2002, p. 160.

21. M. MülLer, Ramakrishna. His life and Sayings, London, 1898.

22. M. MÜLLER, ibid., p. IX.

23. «Le nom neutre brahman est employé dans le Rig-Veda dans le sens de 'prière', originairement ce qui s'échappe de l'âme, et, en un sens, ce qui est révélé. C'est pourquoi brahman est employé plus tard comme nom collectif pour désigner 
analogies entre les notions philosophiques platoniciennes et stoï-

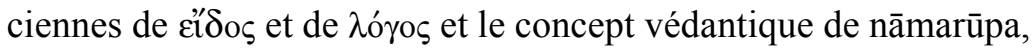
affirmant que l'un comme l'autre renvoient à l'idée de forme et de pensée ou parole. Non seulement la pensée ou parole de Dieu ou de bráhman, pensée et parole étant indifférenciées, serait aux origines de la Création mais encore nom-et-forme représenteraient les pensées manifestes de Dieu, dans le monde créé, grâce auxquelles l'homme pourrait percevoir son propre Créateur. Ne pouvant directement prétendre à identifier étymologiquement le neutre bráhman comme «parole créatrice », Max Müller est obligé d'utiliser un substitut en affirmant que la vāk ou « parole » à laquelle sont dédiés des hymnes rgvédiques devait représenter plus que le simple langage articulé, devenu déité abstraite par la suite. Il s'éloigne ici de l'interprétation naturaliste qu'il donnera quelques années plus tard dans ses Nouvelles études de mythologie, considérant que la vāk « laisse entrevoir au loin comme sa source première la voix de l'orage $»^{24}$. En 1865, Albrecht Weber avait déjà entrepris une étude comparée des notions de vāk et de $\lambda$ ó $\varsigma^{25}$. La vāk rovédique doit donc être comprise, pour Max Müller, comme « une sorte de Logos ou de Sagesse primitive $»^{26}$ lentement divinisée au cours des temps par les brahmanes ${ }^{27}$. Cette vāk aux fonctions cosmogoniques, attestées en RV X.125, aurait, selon lui, un lien étroit avec la hôkmāh de Proverbes 8.22, qu'il donne, suivant la traduction

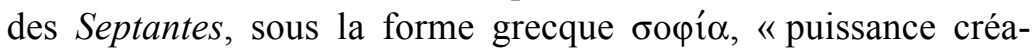

le Veda, la parole sacrée », M. Müller, Essais sur l'histoire des religions, traduction de Georges Harris, Paris, Didier et C $\mathrm{C}^{\mathrm{ie}}$, 1872, n. 1, p. 104.

24. M. Müller, Nouvelle étude de mythologie, in Mythologie comparée, Paris, R. Laffont, 2002, p. 682.

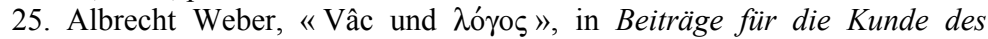
indischen Alterthums, Indische Studien, F.-A. Brockhaus, 1865, p. 473-482.

26. M. Müller, Introduction à la philosophie du védanta, Paris, E. Leroux, 1899, p. 167.

27. «Les brahmanes, dans les hymnes du védas, élevèrent la parole au rang d'une divinité, ainsi qu'ils le faisaient pour toutes les choses dont la nature leur était connue. Ils lui adressaient des hymnes où il est dit qu'elle a habité avec les dieux dès le commencement, accomplissant des choses merveilleuses, et qu'elle n'a jamais été révélée à l'homme si ce n'est en partie. Dans les brahmanas, la parole est appelée la vache, le souffle est appelé le taureau, et l'esprit humain présenté comme leur progéniture. Il est dit que Brahman, le plus grand des êtres, est connu par la parole, et la parole elle-même est appelée la Brahman suprême. », M. Müller, La science du langage, cours professé à l'Institution royale de la Grande-Bretagne en l'année 1861, traduction de G. Harris et G. Perrot, Paris, A. Durand et Pedone-Lauriel, 1867, p. 98-99. 
trice ${ }^{28}$, dont il retrouverait l'écho dans le prologue de l'Evangile

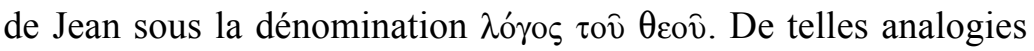
lui permettent non seulement de remonter haut dans le judaïsme ancien mais encore de reconstituer les maillons d'une longue chaîne de transmission d'un concept décliné sous les termes de

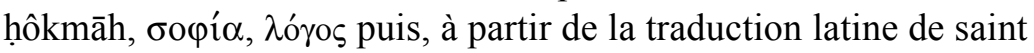
Jérôme, de verbum. Cette mise en parallèle entre la pensée grecque et hébraïque n'est pas nouvelle. Max Müller avait bien des années auparavant déjà émis l'hypothèse que le sens du mot «bráhman, en sanscrit, signifiait originairement " puissance », [et qu']il avait le même sens que El [《 fort $»] »^{29}$. Suivant sa théorie de la maladie du langage, ce bráhman impersonnel aurait été progressivement personnifié et divinisé ${ }^{30}$.

C'est dans les mythes cosmogoniques védiques qu'il va donc rechercher les éléments lui permettant une identification entre vāk et bráhman. Ainsi, en ŚB 6.1.1.8 il prendra la liberté de traduire «prajāpati [...] bráhmaivá prathamám asrjata » par « [Prajāpati] créa tout d'abord le Verbe ${ }^{31}$ considérant qu'il s'agit probablement du sens primitif donné par les ṛși ou «voyants » au neutre bráhman, là où les brahmanes lui substitueront par la suite celui de «Veda » ou de "Savoir sacré » comme l'indique à la suite le commentaire brâhmanique « trayómevá vidyām », « c'est-à-dire la Triple Science ». Étant donné que le « sens original de bráhman peut avoir été oublié $»^{32}$, il conçoit la possible équivalence linguistique entre les composés Bṛhaspati et Vāhaspati qui ne seraient

28. M. Müller, Introduction à la philosophie du védanta, Paris, E. Leroux, 1899 , p. 169.

29. M. Müller, Essais sur l'histoire des religions, traduction de Georges Harris, Paris, Didier et $C^{\text {ie }}, 1872$, p. 494.

30. «Le seul nom qu'il [poète primitif] soit capable de trouver pour cette puissance mystérieuse, c'est Brahman; car brahman a signifié originairement " puissance, volonté, désir, et la force propulsive et créatrice». Mais ce brahman impersonnel, dès qu'il est nommé, devient aussi quelque chose d'extraordinaire et de divin. Il finit par être rangé dans la multitude des dieux, par être un des personnages de la grande triade que l'on adore encore aujourd'hui », M. Müller, ibid., p. 102.

31. M. Müller, Introduction à la philosophie du védanta, Paris, E. Leroux, 1899, p. 172.

32. M. Müller, ibid., p. 172. 
autres que bráhman et qu'il traduit par « Seigneur de la parole » ${ }^{33}$. Il va alors faire valoir les doublets phonétiques des labiales et des labio-dentales des racines brh- et vro- ${ }^{34}$. La racine brh- qu'il prend comme « ce qui éclate dans le sens de création ou créateur, particulièrement quand la création était conçue non comme une fabrication mais comme une expansion ${ }^{35}$, aurait son équivalent en brdh- et vrdh-. De la racine vrdh-, il tire un substantif *vardha- qui « se transformera régulièrement en latin en verbum $»^{36}$. Il note en effet que le sanskrit rudhira se retrouve en latin sous la forme ruber due à l'alternance entre la dentale aspirée sanskrite et la labiale latine. Mais il va encore plus loin. Considérant la chute de l'aspiration de la dentale en anglais selon la loi de Grimm, *vardha qui correspondrait au latin verbum, est dès lors identifiable avec l'anglais word et de conclure :

«brahman, verbum et word procèdent tous de la même racine $v_{o} r h$ ou $v_{o} r d h$, éclater au dehors, et comportent le même sens, c'està-dire mot. Nous ne devons pas conclure aussitôt qu'en conséquence Brahma, comme source de l'Univers, fut tout d'abord conçu comme le Verbe créateur ou le Logos. Cela est trop parfait pour être vrai. Mais le fait que le même mot brahma signifiait le pouvoir créateur qui éclate au dehors, et aussi le mot qui éclate au dehors, peut avoir amené les premiers penseurs de l'Inde à cette idée que la première expansion du monde fut le mot ou la pensée proférée dans et par Brahman. ${ }^{37}$.

En se basant sur le mythe cosmogonique de ŚB 11.2.3, Max Müller montre que bráhman, ayant créé le triple monde mais demeurant au-delà de sa propre création, s'interroge sur le moyen d'y pénétrer. Il y « retourna en utilisant le couple Nom-et-Forme » nous dit le texte védique. Fort de ce constat, il traduit alors un passage de la Maitry upaniṣad (6.22) ainsi : « Deux bráhmans doivent être médités, le verbe et le non-verbe. Ce n'est que par le verbe que le nonverbe est révélé $»^{38}$, là où le texte nous dit «On doit méditer, en

33. M. Müller, ibid., p. 172.

34. Sur ces doublets, voir Louis Renou, Grammaire sanscrite, tomes I et II réunis, $3^{\mathrm{e}}$ édition revue, corrigée et augmentée, Paris, A. Maisonneuve, 1996, § 54, p. 59.

35. M. Müller, ibid., p. 173.

36. M. Müller, ibid., p. 174.

37. M. Müller, ibid., p. 174-175.

38. M. Müller, ibid., p. 178. Il reprend ici la traduction qu'il avait déjà donnée dix ans auparavant: "Two Brahmans have to be meditated on, the word and 
vérité, sur deux bráhman : celui qui est le son et celui qui est le non-son. C'est par le son que le non-son est manifesté. Grâce à Oṃ, le son s'échappe vers le haut et va se perdre dans le non-son. Voilà en vérité quelle est la voie; voilà l'immortalité, voilà l'union (au bráhman), voilà la béatitude $»^{39}$. L'upaniṣad enseigne que le son Om identifié à l'ātman, n'étant autre que le bráhman manifesté dans sa propre création, va rejoindre le non-son, bráhman, assurant à « celui qui sait ainsi » la délivrance. Nous attendrions ici que Max Müller argumente le choix de sa traduction du sanskrit śabda et aśabda, « son » et « non-son », par « word » et « non-word ». Là où il ne peut plus trouver d'équivalences étymologiques entre śabda et bráhman, il charge sémantiquement le premier terme de la valeur qu'il a attribuée dans sa démonstration au second sans plus la justifier. Passant sous silence cette traduction forcée et orientée, Max Müller en déduit :

«Ici, nous avons de nouveau la contre-partie exacte du Logos de l'école d'Alexandrie. Suivant cette dernière, l'Essence divine est révélée par le Verbe et par le Verbe seul. En son état irrévélé, elle est inconnue, et quelques philosophes chrétiens l'appelaient le Père; en son état révélé elle était le Logos divin ou le Fils. De tout ce qui précède, il me semble que nous sommes amenés à admettre que la même suite d'idées qui, après une longue préparation, trouva son expression définitive dans Philon et plus tard dans Clément d'Alexandrie, fut élaborée dans l'Inde à une époque plus reculée, en partant de débuts très semblables pour arriver à des résultats presque identiques. Mais rien ne démontre qu'il y ait eu emprunt d'un côté ou de l'autre $»^{40}$.

N'est-ce pas pourtant le même Max Müller qui en 1879, quelque quatorze années auparavant, affirmait dans une note relative à l'étymologie et à la définition de ce même neutre bráhman que :

«sans essayer de suivre les dernières ramifications de brahman, parole, hymne d'éloge, prière, sacrifice, je me contenterai de mettre en garde contre l'idée que nous aurions là une sorte de Logos. Bien qu'il

the non-word. By the word alone is the non-word revealed. Now there is the word Om. Moving upward by it (where all words and all what is mean t by them ceases), he arrives at absorption in the non-word (Brahman). This is the way, this is the immortal, this is union, and this is bliss", The Upanishads translated by F. Max Müller, part II, Oxford, The Clarendon Press, 1884, p. 321.

39. Maitry Upanishad, trad. Anne-Marie Esnoul, Paris, A. Maisonneuve, 1952.

40. M. Müller, Introduction à la philosophie du védanta, Paris, E. Leroux, 1899, p. $178-179$. 
finisse par désigner la cause de l'univers et soit fréquemment identifié avec l'Atman, l'âme suprême, son développement est différent de celui du Logos alexandrin, et en tout cas, au point de vue historique, il y a là deux courants absolument indépendants $\gg{ }^{41}$.

Force est de constater que le rapprochement opéré par Max Müller entre la philosophie grecque et le vedānta repose bien plus, voire entièrement, sur l'étymologie que sur l'étude sémantique des notions philosophiques. Résoudre l'équation bráhman $=$ verbum était certainement pour ce grand savant dépasser cette découverte qu'il considérait comme la plus importante de son siècle : Zeus = Dyaus. C'était démontrer qu'au-delà des correspondances morphologiques et des modifications phonétiques suivant la loi de Grimm, les mots eux-mêmes renfermaient un sens identique exprimant la pensée primitive de ces lointains ancêtres indo-européens au sujet de ce qu'ils percevaient comme source première du monde phénoménal. Malgré des avancées certaines dans le domaine des études sanskrites au cours du $\mathrm{xx}^{\mathrm{e}}$ siècle, le terme bráhman a toujours posé bien des interrogations quant à son étymologie ${ }^{42}$. Louis Renou a émis l'hypothèse d'un possible rapprochement du substantif avec une « racine *brah- / *barh- au sens de 'parler par énigmes', qui rendrait compte de bráhman » en tant qu' « énigme » ou «formule énigmatique $\gg^{43}$.

\section{Pour Conclure}

La démonstration de Max Müller n'est pas sans intérêt. Elle a le mérite de porter notre attention sur la spéculation upanișadique qui peut, par certains côtés, être rapprochée de la philosophie néoplatonicienne et pose également la question des nature et fonction attribuées au langage, parole et pensée, au sein même des mythes cosmogoniques. De nombreuses études ultérieures se sont d'ailleurs intéressées à de telles convergences entre les pensées

41. M. Müller, Origine et développement de la religion étudiés à la lumière des religions de l'Inde, Paris, C. Reinwald, 1879, n. 1, p. 322.

42. Jan Gonda, Les religions de l'Inde, vol. I : Védisme et hindouisme ancien, Paris, Payot, 1962, p. 45-46 et la note 3, p. 45.

43. L. Renou, "Sur la notion de Bráhman », in L'Inde fondamentale, Paris, Hermann, 1978, p. 94-95. 
grecque et indienne et demeurent toujours d'actualité ${ }^{44}$. Mais, en matière de comparatisme, la recherche étymologique s'avère peu convaincante car elle fait fi de la portée sémantique dont se charge chacun des termes (bráhman, $\lambda$ ó ${ }^{\circ} \varsigma$, verbum) dans son contexte philosophique ou théologique de son temps. Comme l'a si justement rappelé François Chenet au sujet de la méthode comparative appliquée aux philosophies grecques et indiennes, « il serait dangereux et tout à fait illusoire de classer sous des rubriques portant des noms d'origine grecque ou latine des notions, des enchaînements d'idées ou des philophèmes appartenant à une culture aussi différente de la nôtre $»^{45}$. Ce fut le cas de Max Müller qui passait outre toute approche historico-critique et visait avant tout par l'étymologie à démontrer qu'en un temps reculé ces termes bráhman, verbum et word eurent le même sens. Or, comment pourrait-on rapprocher bráhman du vieil anglais word dérivant du gothique waurd et dont on trouve des correspondances en avestique urvata-, en sanskrit vrata-, en grec $\rho ́ \eta ́ \tau \rho \alpha$ ou en hittite weriga et remontant à un hypothétique indo-européen *were exprimant «l'ordre donné », «la parole de commandement»? Si les équivalences morphologiques et phonétiques peuvent être acceptées entre verbum et word, elles ne le peuvent guère avec bráhman. La recherche étymologique ne peut guère en ce domaine tout expliquer et donner le sens exact des termes philosophiques employés dans des milieux intellectuels bien spécifiques. Les mots choisis pour exprimer des notions abstraites ont été chargés, par chacun des courants philosophiques, de signifiés bien particuliers qui ne peuvent être saisis qu'en étudiant ceux-ci dans leur propre contexte. En ce cas, l'étymologie, et plus généralement la philologie, doit céder le pas ou recourir à la méthode historico-critique. Il est intéressant de compulser les traductions sanskrites des livres bibliques afin de voir comment les chrétiens, aidés parfois de pandits indiens, ont essayé au cours de ce même

44. Kenneth Saunders, The Eternal Order : Logos, Brahman and Dharma, Kessinger Publishing, 2007.

45. François Chenet, « Du sens de la philosophie comparée », in Philosophie comparée, Grèce, Inde, Chine, coordination scientifique Joachim Lacrosse, Paris, J. Vrin, 2005, p. 85. Certaines thèses avancées dans cet ouvrage illustrent parfaitement les limites et les défaillances de toute démarche comparative lorsque celle-ci n'est pas étayée par une solide approche historique des milieux philosophiques étudiés et ne diffèrent finalement en rien des rapprochements analogiques faits par Max Müller plus d'un siècle auparavant. 
$\mathrm{XIX}^{\mathrm{e}}$ siècle de retranscrire les idées fondamentales des textes judéochrétiens. Nous constatons que la notion de hôkmāh en Proverbes 8.22 a été rendue par le sanskrit buddhi ${ }^{46}$ et que le $\lambda$ ó $\gamma$ os du prologue de l'Evangile de Jean a été traduit soit par vācana ${ }^{47}$ soit par vāda ${ }^{48}$. Dans chacun des cas, les traducteurs chrétiens ne se sont pas référés à la vāc telle qu'elle est définie dans les traités upaniṣadiques et par la pensée védantique. Les équivalences étymologiques n'ont pas ici été recherchées, ni les hypothétiques liens possibles entre la pensée chrétienne grecque et la philosophie védantique. Les missionnaires chrétiens ont donc tenté avant tout de rendre en sanskrit la portée sémantique des concepts définissant le fondement de leur propre tradition religieuse en en conservant ainsi toute la spécificité et en évitant ainsi tout risque de syncrétisme. C'est cette même spécificité que l'historien des religions doit, avant toute interprétation, s'efforcer de déterminer au mieux lorsqu'il aborde les concepts, les termes techniques, employés par des doctrines religieuses d'un temps autre que le sien et d'une culture autre que la sienne.

Si le $\lambda$ ó ${ }^{\circ} \varsigma \varsigma$ ou bráhman est apparu aux yeux de Max Müller comme un pont jeté entre Infini et Fini, entre Divin et Humain ${ }^{49}$, il fut aussi un pont jeté entre Orient et Occident, entre religions dont les fervents défenseurs auraient dû prendre conscience, en cette fin

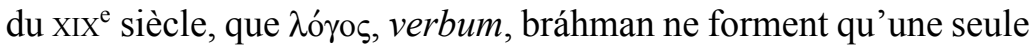
et même réalité. C'est peut être là toute la force de son humanisme et de sa mystique. Celui qui fut influencé par l'enseignement de Maître J. Eckhart et des mystiques rhénans avait trouvé dans le vedānta la confirmation de l'universalité de la présence de Dieu en l'homme et souhaitait en témoigner par une approche scientifique à l'humanité, voire même, avant de se fondre dans l'Infini pour l'éternité, lui proposer une religion nouvelle à l'aube du $\mathrm{xx}^{\mathrm{e}}$ siècle. En parlant

46. The Holy Bible Translated into the Hinduee Language by the Reverend William Bowley under the Patronage of the Calcutta Auxiliary Bible Society, vol. II, I Chronicles to Malachi, 1834, p. 11.

47. The New Testament of our Lord and Saviour Jesus Christ Translated from the Original Greek, North India Bible Society, Secundra Orphan Press, 1849, p. 235.

48. ādau vāda āsīt, sa ca vāda î́varābhimukha āsīt, sa ca vāda î́vara āsīt, The New Testament in Sanskrit, 1910, p. 215.

49. M. Müller, Theosophy or Psychological Religion, the Gifford Lectures, London, Longmans \& Green, 1893, p. 361s. 
devant les membres de l'Institut Royal de Grande-Bretagne, Max Müller n'aura peut-être finalement pas trouvé mieux pour remplir le contrat qu'il s'était donné de leur démontrer que, bráhman, l'un des termes fondamentaux de la philosophie du vedānta, issue alors d'une des nombreuses colonies de la Couronne, avait des liens au moins étymologiques avec la langue anglaise et ainsi parvenir à son but qu'il expose dès l'introduction de sa première conférence : « Je ne me dissimule pas les difficultés que je rencontrerai en essayant de gagner votre intérêt, bien plus, s'il est possible, votre sympathie, en faveur d'un antique système de philosophie hindoue, la philosophie védanta $»^{50}$.

InSTITUT D'Histoire DES ReLigions

UFR des Sciences Historiques

Université de Strasbourg

9, place de l'Université

67084 Strasbourg Cedex

50. M. Müller, Introduction à la philosophie du védanta, Paris, E. Leroux, 1899, p. 1. 